\title{
Drug-Coated Balloon vs. Stent for de novo Non-small Coronary Artery Disease: A Systematic Review and Meta-Analysis
}

\author{
Kaiwen Sun ${ }^{1}$, Zhenzhu Liu ${ }^{2}$ and Hongyan Wang ${ }^{2 *}$ \\ ${ }^{1}$ The Second Hospital of Dalian Medical University, Dalian Medical University, Dalian, China, ${ }^{2}$ Department of Cardiovascular \\ Medicine, The Second Hospital of Dalian Medical University, Dalian, China
}

Introduction: Drug-coated balloon (DCB) has been an attractive option in de novo vessels. A systematic review and meta-analysis were conducted to evaluate the efficacy and safety of DCB vs. stent for treating de novo lesions in non-small vessels.

Methods: Studies in PubMed, Embase, the Cochrane Central Register of Controlled Trials, and Web of Science were searched (from their commencement to March 2021). This meta-analysis was performed by Review Manager 5.3.

OPEN ACCESS

Edited by:

Tommaso Gori,

Johannes Gutenberg University

Mainz, Germany

Reviewed by:

Mariama Akodad,

Centre Hospitalier Universitaire de

Montpellier, France

Alessandro Sticchi,

Bern University Hospital, Switzerland

${ }^{*}$ Correspondence:

Hongyan Wang

2577223926@qq.com

Specialty section: This article was submitted to

Coronary Artery Disease,

a section of the journal

Frontiers in Cardiovascular Medicine

Received: 25 April 2021 Accepted: 09 November 2021

Published: 10 December 2021

Citation:

Sun K, Liu Z and Wang H (2021) Drug-Coated Balloon vs. Stent for de

novo Non-small Coronary Artery

Disease: A Systematic Review and Meta-Analysis.

Front. Cardiovasc. Med. 8:700235.

doi: 10.3389/fcrm.2021.700235
Results: A total of 3 random controlled trials (RCTs) with 255 patients and 2 observational studies (OS) with 265 patients were included in this meta-analysis following our inclusion criteria. It could be observed that DCB presented no significant difference in cardiac death (CD) (RR 0.33, 95\% Cl [0.01, 8.29], $p=0.50$ in OS), myocardial infarction (MI) (RR 0.49, 95\% Cl [0.09, 2.50], $p=0.39$ in $\mathrm{RCT})$, target lesion revascularization (TLR) $(R R 0.64,95 \% \mathrm{Cl}[0.19,2.18], p=0.47$ in RCT) $(R R 1.72,95 \% \mathrm{Cl}[0.56,5.26], p=0.34$ in OS), and late lumen loss (LLL) (SMD $-0.48,95 \% \mathrm{Cl}[-1.32,0.36], p=0.26$ in RCT) for de novo non-small coronary artery disease (CAD) compared with stents, whereas minimal lumen diameter (MLD) including MLD1 (SMD -0.67, 95\% Cl [-0.92 -0.42], $p$ $<0.00001$ in RCT) and MLD2 (SMD $-0.36,95 \% \mathrm{Cl}[-0.61-0.11], p=0.004$ in RCT) was smaller in DCB group.

Conclusion: This systematic review showed that DCB might provide a promising way on de novo non-small coronary artery disease compared with stents. However, more RCTs are still needed to further prove the benefits of the DCB strategy.

Systematic Review Registration: https://www.crd.york.ac.uk/PROSPERO/\# recordDetails.

Keywords: drug-coated balloon, de novo vessels, non-small, stent, systematic review

\section{INTRODUCTION}

Coronary artery disease (CAD) is still the leading cause of human death and disability worldwide according to the latest epidemiological data from the Global Burden of Disease (GBD) (1). Percutaneous coronary intervention (PCI) is a therapeutic intervention by expanding the stenosis or occlusion of the vascular lumen, which improves the circulation of myocardium. With the development of technology, PCI has been applied to more complex lesions among patients with 
CAD $(2,3)$. At present, most of the PCI is expanding the target lesions with stents, from bare metal stent (BMS) to firstgeneration drug-eluting stent (DES) and second- or newergeneration DES, which can not only significantly reduce the incidence and mortality of myocardial infarction (MI) in highrisk patients with massive myocardial ischemia, but also improve the quality of life of patients. Despite the superiority of stents in the management of CAD, very late adverse cardiovascular events still exist after stent implantation (4). Though with the advent and application of DES, complications such as in-stent restenosis (ISR) and stent thrombosis still occur, leading to the recurrence of angina or acute coronary syndrome (ACS) $(5,6)$. There are also technical challenges in the application of stents in complex lesions, which affect its long-term efficacy. A recent study reported a new complication of longitudinal stent deformation (LSD), which is defined as the distortion or shortening of intracoronary stents after successful stent deployment because of the reduced longitudinal strength of stents (7). There are also some rare complications such as spontaneous coronary artery pseudo aneurysm and infection according to the report $(8,9)$.

In recent years, drug-coated balloon (DCB) has been investigated and applied in clinical for its no residues. It has become 1 of the preferred options for the treatment of ISR by the ESC guideline (10). There are also many potential benefits such as de novo vessel disease and patients with a high risk of bleeding. In addition, there are other potential indications, such as bifurcation lesions, chronic total occlusion lesions, and others (11-13). With the advantage of DCB, there are some systematic reviews and meta-analysis based on the role of DCB in de novo vessels, whose results showed that $\mathrm{DCB}$ was noninferior to the stent strategy when treating the target lesions (14-16). However, those results were concentrated more on de novo small vessels or unclassified vessel diameter of de novo vessels. Besides, those results included BMS as a control, which has been rarely applied and not recommended for PCI by the recent guidelines. With the development and advance of DCB, there were several studies that used DCB for treating with de novo non-small vessels (1721). Lin et al. (22) did a meta-analysis in de novo large vessels, though remained some limitations. First, only 1 study with stable CAD was included in their study and the subgroup analysis was limited. Second, the end points in their study were not enough since there were more clinical and angiographic outcomes which could be used to assess the efficacy and safety of DCB. Finally, the number of studies was not enough and contained 1 nonrandom controlled trial (RCT).

Therefore, we conducted a systematic review and metaanalysis to evaluate the efficacy and safety of DCB vs. stent for treating de novo lesions in non-small vessels.

\section{MATERIALS AND METHODS}

\section{Protocol}

This meta-analysis followed the Preferred Reporting Items for Systematic Meta-Analysis (PRISMA) statement (23). The protocol was registered at PROSPERO (registration number: CRD42021244832).

\section{Search Strategy}

The following electronic databases were searched: PubMed, Embase, the Cochrane Central Register of Controlled Trials, and Web of Science from their commencement to March 2021 for the relevant literature. A manual search of references was performed for additional omitted studies. The specific search strategies were presented in the supplemental.

\section{Selection Criteria}

The literature was independently screened and evaluated by 2 authors (Sun and Liu). In total, 2 authors scanned the title and abstract according to the inclusion criteria and then screened again by reading the full text. If there were any disagreements, it would be discussed with another author (Wang) and resolved by consensus, or contacted the original author of the article if necessary.

The inclusion criteria were as follows: (1) patients with CAD; (2) PCI for de novo lesions with lumen diameters $\geq 2.5 \mathrm{~mm}$; (3) DCB as experimental group without any restriction on the type of DCB; (4) stent as control group for DES only; and (5) studies included RCT and observational study (OS).

The exclusion criteria were as follows: (1) patients with peripheral vascular diseases; (2) PCI for de novo lesions with lumen diameters $<2.5 \mathrm{~mm}$; (3) studies were reviewed or conference abstract; and (4) data inefficient.

\section{Study Outcomes and Definition}

The study outcomes could be divided into clinical outcomes and angiographic outcomes. The clinical outcomes included cardiac death (CD), MI, and target lesion revascularization (TLR). CD was defined as death caused by cardiac factors. MI was defined based on the latest ESC guidelines (10). TLR was defined as any repeat revascularization due to restenosis in treated segments including $5 \mathrm{~mm}$ proximally and distally. The angiographic outcomes included minimal lumen diameter (MLD), which was divided into MLD1 (MLD immediately after PCI) and MLD2 (MLD at follow-up angiography), and late lumen loss (LLL), which means MLD1 minus MLD2. The angiographic outcomes were obtained by quantitative coronary angiography (QCA).

\section{Data Extraction}

The following data were extracted from eligible studies independently by 2 authors (Sun and Liu): the study of the author, year, and country; patients' indication; the number of patients, age, and sex in each group; the vessel diameter and length; the main characteristics of DCB; the type of DCB and stents; lesion preparation for DCB and stents; clinical and angiographic outcomes and follow-ups.

\section{Quality Assessment}

The quality of the eligible RCT studies and the risk of bias were assessed by 2 independent authors (Sun and Liu) using the Cochrane Handbook for Systematic Reviews of Interventions, version 5.1.0, which contains seven criteria: random sequence generation, allocation concealment, blinding of participants and personnel, blinding of outcome assessment, incomplete outcome 
data, selective outcome reporting, and other bias. These seven criteria were rated as "low risk," "unclear risk," or "high risk" depending on the characteristics of each criterion reported in the study. For OS, the quality of the studies was assessed by the Newcastle-Ottawa Scale (NOS) with 8 items including selection, comparability, and outcome. A study with more than 6 stars was regarded as a high-quality one.

\section{Statistical Analysis}

The effect of DCB or stents for de novo non-small vessels was measured by relative risks $(R R)$ for all clinical outcomes and standard mean difference (SMD) for all angiographic outcomes. As several included studies did not offer the changes in the standard deviation (SD) of LLL or MLD from the baseline values, we calculated the SD change by the following formula (1). The coefficient was taken as 0.5 according to other eligible studies that provided the SD baseline, SD final, and SD change.

$\mathrm{SD}$ change $=\sqrt{ }\left[(\mathrm{SD} \text { pretreatment })^{2}+(\mathrm{SD} \text { posttreatment })^{2}\right.$

- (2* coefficient $*$ SD pretreatment $*$ SD posttreatment $)](1)$

This meta-analysis was performed by Review Manager 5.3 (Cochrane Collaboration, 2014). Heterogeneity of the studies

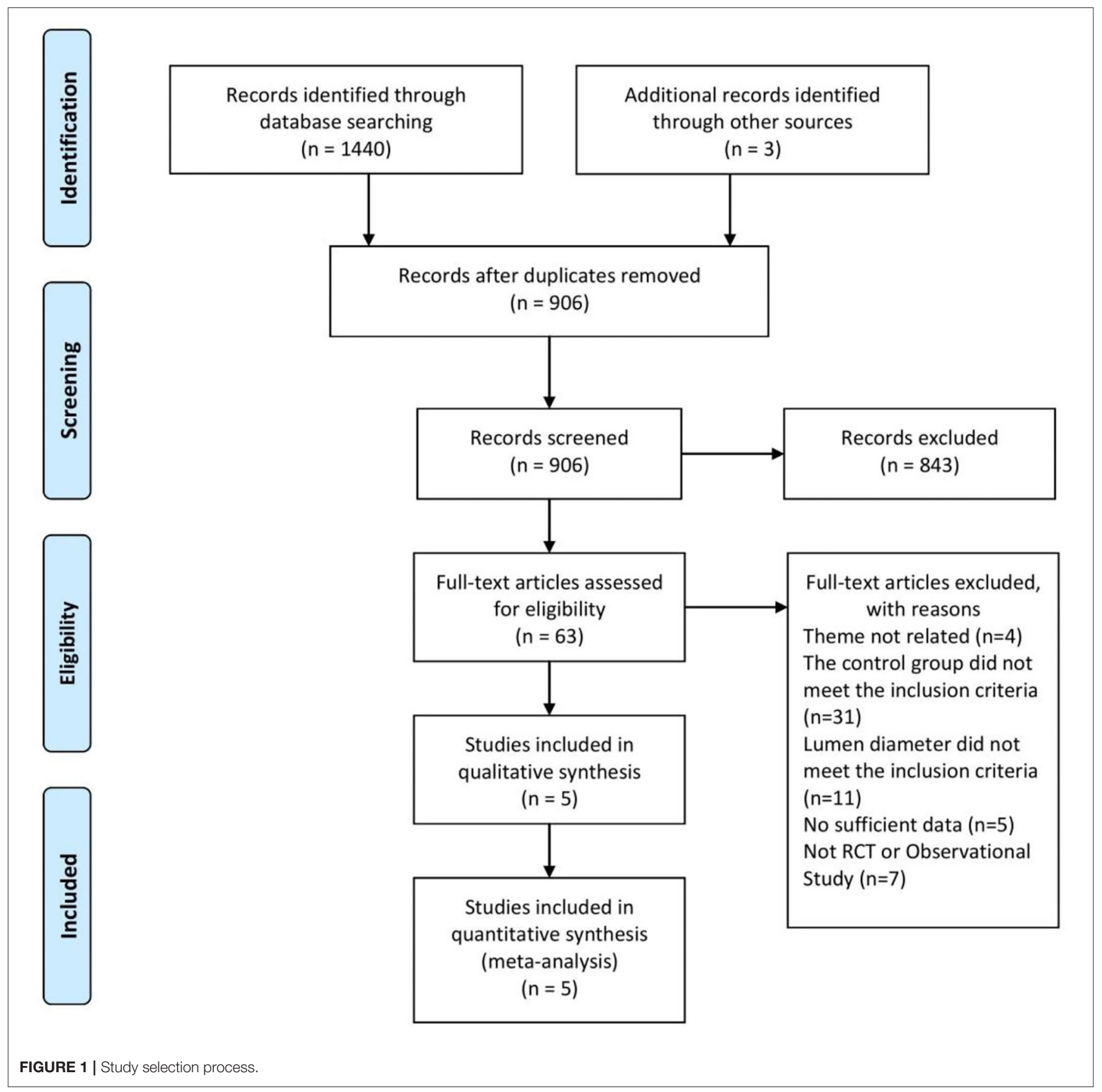


was assessed by the Cochran's Q statistic and $I^{2}$ test. Studies were considered homogeneous if the $p$-value of the Q-test was $>0.1$ or the $I^{2}$ value was $<50 \%$, or it would be considered as heterogeneity. A random effect model was chosen to analyze the data.

To further explore the other factors associated with the results, a series of subgroup analyses were performed by Review Manager 5.3 (Cochrane Collaboration, 2014), including the type of patient status, age, the type of $\mathrm{DCB}$, and the clinical or angiographic follow-up, with $p<0.05$ considered statistically significant. The sensitivity analysis was done using the leave-one-out method to examine the stability of the results.

\section{RESULTS}

\section{Description of Studies}

A total of 1,443 studies were retrieved based on the search terms and strategies described ahead. There had been 906 studies to be screened by titles and abstracts since 843 studies were removed because of repetition. Of the 906 studies, 63 studies remained to be read the full text for further exclusion. Finally, 5 studies (17-21), including 3 RCTs and 2 OS, were selected and included in this meta-analysis with the inclusion criteria (Figure 1). The characteristics of included study were summarized in the Table $\mathbf{1}$ as shown below.

\section{Quality Assessment}

The Cochrane risk of bias tool was used to assess the methodological quality of the included RCT studies, as were presented in the Figures 2, 3. For selection bias, most studies showed a low risk whereas 2 studies $(17,19)$ did not specifically describe allocation concealment. For performance bias, 1 study (17) did not take any blind methods, which might have an effect on the results. For detection bias, all studies could explain the results despite the blind method. In terms of attrition bias and reporting bias, 2 studies $(18,19)$ failed to provide comprehensive information on follow-up or exclusion. For reporting bias and other bias, no study presented any obvious one. The NOS was used to assess the quality of OS from selection, comparability, and outcome. Both the 2 OS were of a high quality by rewarding 8 and 7 stars which were shown in Table 2.

\section{Clinical and Angiographic Outcomes $\mathrm{CD}$ and $\mathrm{MI}$}

Of the 3 RCTs and 2 OS, CD was observed in only 1 study (20), with no significant difference between the DCB and stents $(R R$ $0.33,95 \%$ CI $[0.01,8.29], p=0.50)$. MI was observed in only 1 study (18), with no significant difference between the DCB and stents $(R R 0.49,95 \%$ CI $[0.09,2.50], p=0.39$ ) either. The specific description results were presented in the Figures 4, 5 .

\section{TLR}

In RCT, the results showed no significant difference between DCB and stents in the rate of TLR (RR 0.64, 95\% CI $[0.19,2.18]$, $p=0.47)$. In OS, there was no significant difference in the rate of TLR $(R R 1.72,95 \%$ CI $[0.56,5.26], p=0.34)$ either. There was lower heterogeneity in $\operatorname{RCT}\left(I^{2}=0, p=0.48\right)$ and $\mathrm{OS}\left(I^{2}=0\right.$, 


\section{Random sequence generation (selection bias)}

Allocation concealment (selection bias)

Blinding of participants and personnel (performance bias)

Blinding of outcome assessment (detection bias)
Incomplete outcome data (attrition bias)
Selective reporting (reporting bias)
Other bias

Blinding of outcome assessment (detection bias)
Incomplete outcome data (attrition bias)
Selective reporting (reporting bias)
Other bias

Blinding of outcome assessment (detection bias)
Incomplete outcome data (attrition bias)
Selective reporting (reporting bias)
Other bias
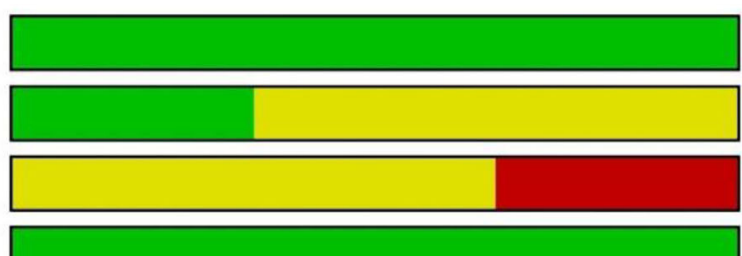

Other bias
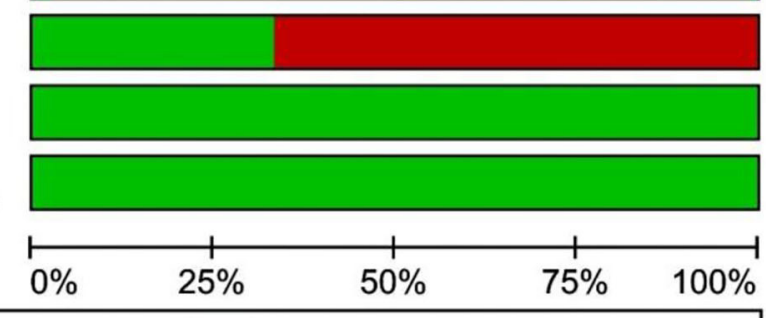

Low risk of bias

Unclear risk of bias

High risk of bias

FIGURE 2 | Risk of bias graph review authors' judgments about each risk of bias item presented as percentages across all included RCT.

$p=0.67)$. The specific description results were presented in the Figure 6.

\section{LLL}

In RCT, the results showed no significant difference between DCB and stents with significant heterogeneity (SMD - 0.48, 95\% CI $\left.[-1.32,0.36], p=0.26, I^{2}=90 \%, p<0.0001\right)$. In OS, only 1 study (21) provided the angiographic outcomes, which could not be calculated the combined effect size (CES) and thus it was excluded. The specific description results were presented in the Figure 7.

\section{MLD1}

In RCT, the results showed that MLD1 in DCB group was smaller than stent group with lower heterogeneity (SMD $-0.67,95 \%$ CI $\left.[-0.92-0.42], p<0.00001, I^{2}=0, p=0.70\right)$. In OS, only 1 study (21) provided the angiographic outcomes, which could not be calculated the CES and thus it was excluded. The specific description results were presented in the Figure 8.

\section{MLD2}

In RCT, the results showed that MLD2 in DCB group was smaller than stent group with lower heterogeneity (SMD -0.36, 95\% CI $\left.[-0.61-0.11], p=0.004, I^{2}=0, p=0.97\right)$. In OS, only 1 study (21) provided the angiographic outcomes, which could not be calculated the CES and thus it was excluded. The specific description results were presented in the Figure 9.

\section{Subgroup and Sensitivity Analysis}

Subgroup analyses were performed to evaluate the effect of type of patient status, age, the type of DCB, and the clinical or angiographic follow-up in TLR, LLL, and MLD. For TLR, there was no significant difference between DCB and stents in the subgroups. For LLL, there was still a high heterogeneity in most subgroups though the heterogeneity was lower when the followup was more than 6 months and the results showed no significant difference. For MLD, it could be observed that MLD1 and MLD2 in DCB group were smaller in each subgroup. The detailed description results were shown in the form below (Tables 3-6).

To explore the impact of each study on the stability of the combined results, a sensitivity analysis was conducted using the one-study removed approach. The heterogeneity and the CES of TLR ( $R R$ 0.64, 95\% CI $[0.19,2.18])$, MLD1 (SMD - 0.67, 95\% CI $[-0.92-0.42])$, and MLD2 (SMD - 0.36, 95\% CI [-0.61 -0.11]) were not significantly changed, which indicated the results were robust enough. We observed that the heterogeneity of LLL was declined ( $I^{2}$ from $90 \%$ turned to $58 \%$ ) when the study of Gobic et al. (18) was removed, whereas the CES (SMD -0.48, 95\% CI $[-1.32,0.36])$ was not significantly changed.

\section{DISCUSSION}

This systematic review and meta-analysis included 3 RCTs and 2 OS following the inclusion criteria. The results indicated that DCB presented no significant difference in CD, MI, TLR, and LLL for de novo non-small CAD compared with stents, whereas MLD1 and MLD2 were smaller in DCB group. We performed the subgroup analysis, which could be observed that MLD1 and MLD2 were also smaller. Thus, these data indicated that DCB might provide a promising way for de novo non-small CAD.

There had been studies on the comparison between DCB and DES or other types of stents, but their data only focused on the small vessels or did not consider the vessel diameter of de novo lesions. Lin et al. (22)suggested that DCB appeared to be an interventional and stentless alternative for treating de novo coronary lesions in large vessels, though there were still some limitations left as described before. Thus, this systematic review and meta-analysis were conducted to evaluate DCB vs. 


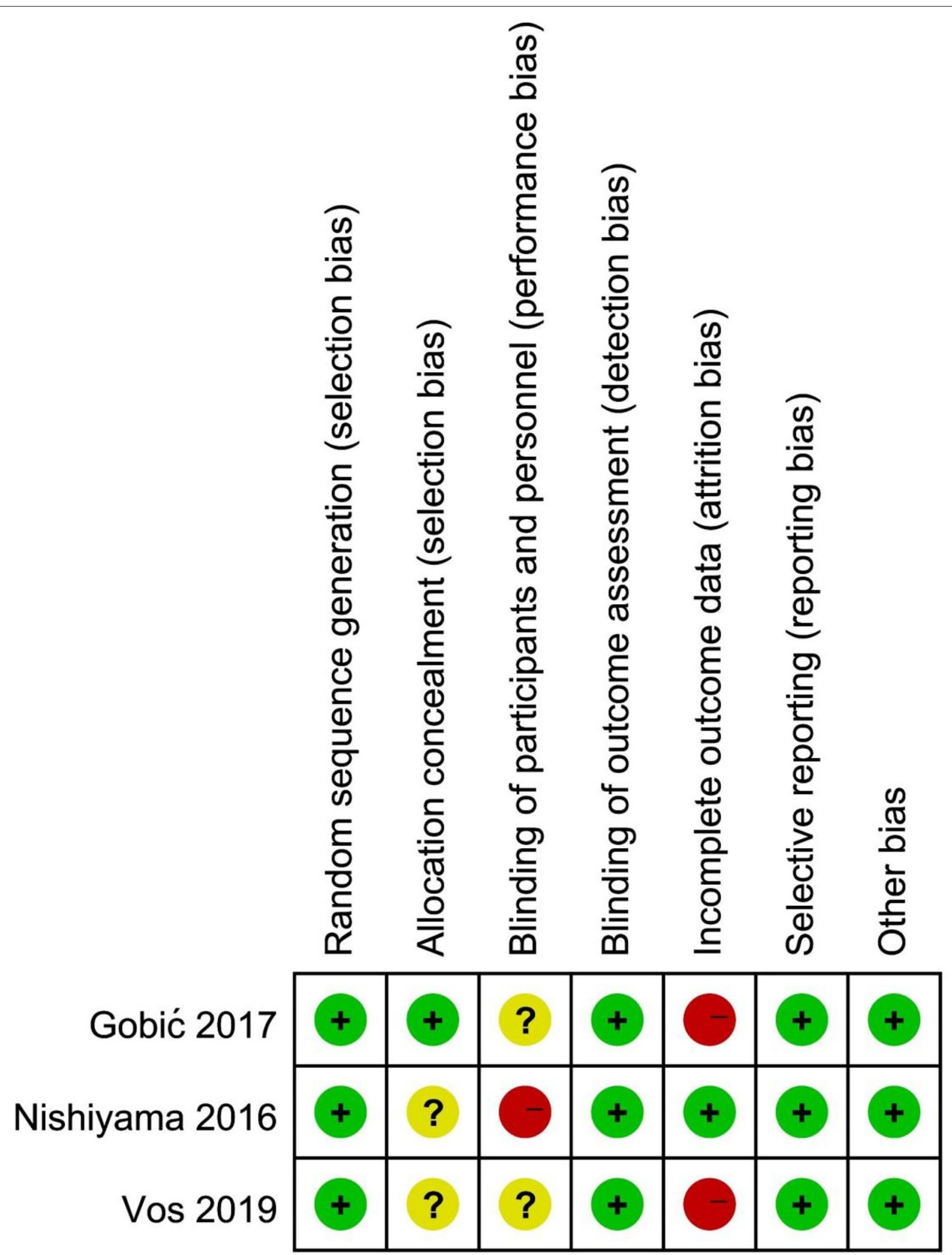

FIGURE 3 | Risk of bias summary review authors' judgements about each risk of bias item for each included RCT. 
stent for treating de novo lesions in non-small vessels. Before the implantation of DCB or stent, lesion preparation was introduced to improve the procedure success rate and reduce late adverse events. Balloon dilation is the most common way and has been widely applied for lesion preparation. Patients with STEMI would be considered adding for thrombus aspiration. However, it remains potential complications including suboptimal dilatation, dissections, plaque shift, elastic recoil, and vessel perforation with high-pressure balloon inflation (24). Iwasaki et al. (21) chose rotational atherectomy which has been demonstrated a preferred choice in calcified lesions in both $\mathrm{DCB}$ and stent group for balloon or stent delivery and expansion, as it could reduce calcified plaque. For DCB, lesion preparation might be more important without stent implantations due to elastic recoil and dissections. Recently, scoring balloon has presented an interventional tool for better plaque modification and improved stent or balloon expansion in lesion preparation. Nonslip element (NSE) balloon is a novel scoring balloon as used in Nishiyama et al. (17). It is similar to cutting balloon and can partially prevent elastic recoil and limit traumatic vascular wall injury. According to the recent study (25), NSE balloon was proved safe and effective in the treatment of de novo CAD combined with DCB. The clinical outcomes including CD, MI, and TLR and the angiographic outcomes including LLL, MLD1, and MLD2 were brought to assess the efficacy and safety of DCB. In this study, CD could be explained as death caused by cardiac factors and MI was defined by the latest ESC guidelines (10). TLR was defined as any repeat revascularization due to restenosis in treated segments including $5 \mathrm{~mm}$ proximally and distally. We retrospected the included study and found the definitions of clinical outcomes in each study met our criteria. For angiographic outcomes, both LLL and MLD were defined and measured by QCA according to the included study (17-19). From the eligible studies included in this meta-analysis, only Her et al. (20) found CD events, and MI events were found in Gobic et al. studies (18). Thus, the subgroup analysis of CD and MI was abandoned because of studies which is not enough. For TLR, there was no significant difference between the DCB and stent group in RCT and OS. No significant difference was observed in subgroup analysis either. The precise quantification of luminal narrowing had been a challenge for a long time before, and it was solved since percutaneous transluminal coronary angioplasty (PTCA) by Andreas Grüntzig in 1977 was generated, which was also the greatest incentive to the development of QCA. QCA has played an important part in evaluating interventional techniques and assessing the results of DCB and stents $(26,27)$. QCA metrics including LLL and MLD (including MLD1 and MLD2) were chosen as angiographic outcomes in this study. Since Her et al. (20) did not provide angiographic outcomes, the results of OS were excluded as only 1 OS could not be calculated the CES and not convincing enough. Thus, 3 RCTs (17-19) were included to explore the angiographic outcomes. For MLD1, it could be observed that MLD1 was smaller in DCB group than stent group in RCT. It presented similar results in MLD2. The subgroup analysis showed that both MLD1 and MLD2 were smaller in each subgroup. MLD in this study was at postprocedural and follow-up, and it should be considered with the preprocedural MLD together.

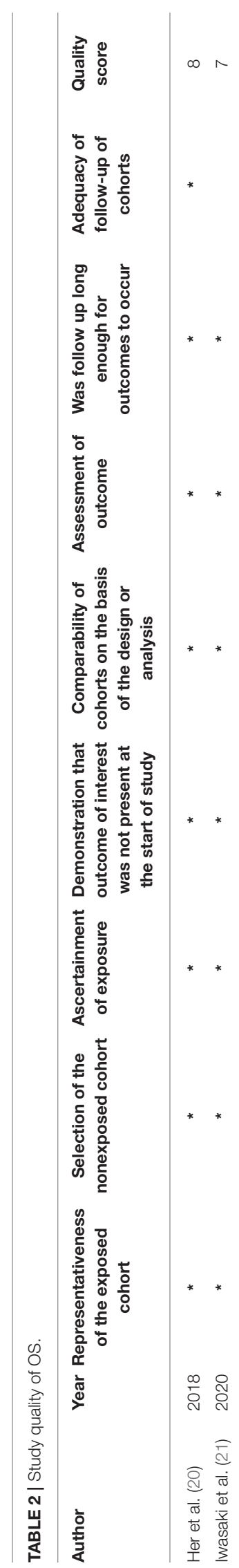




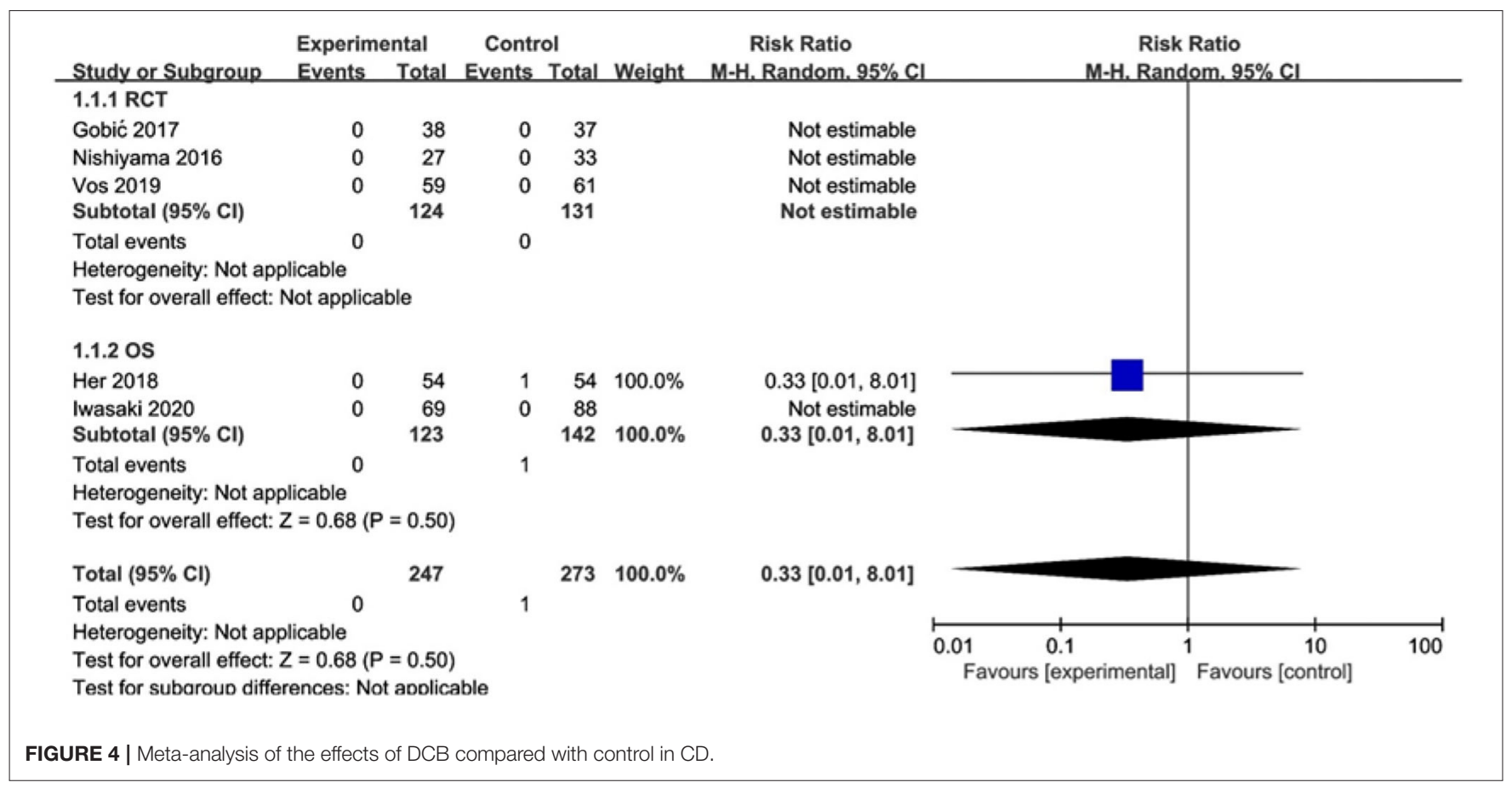

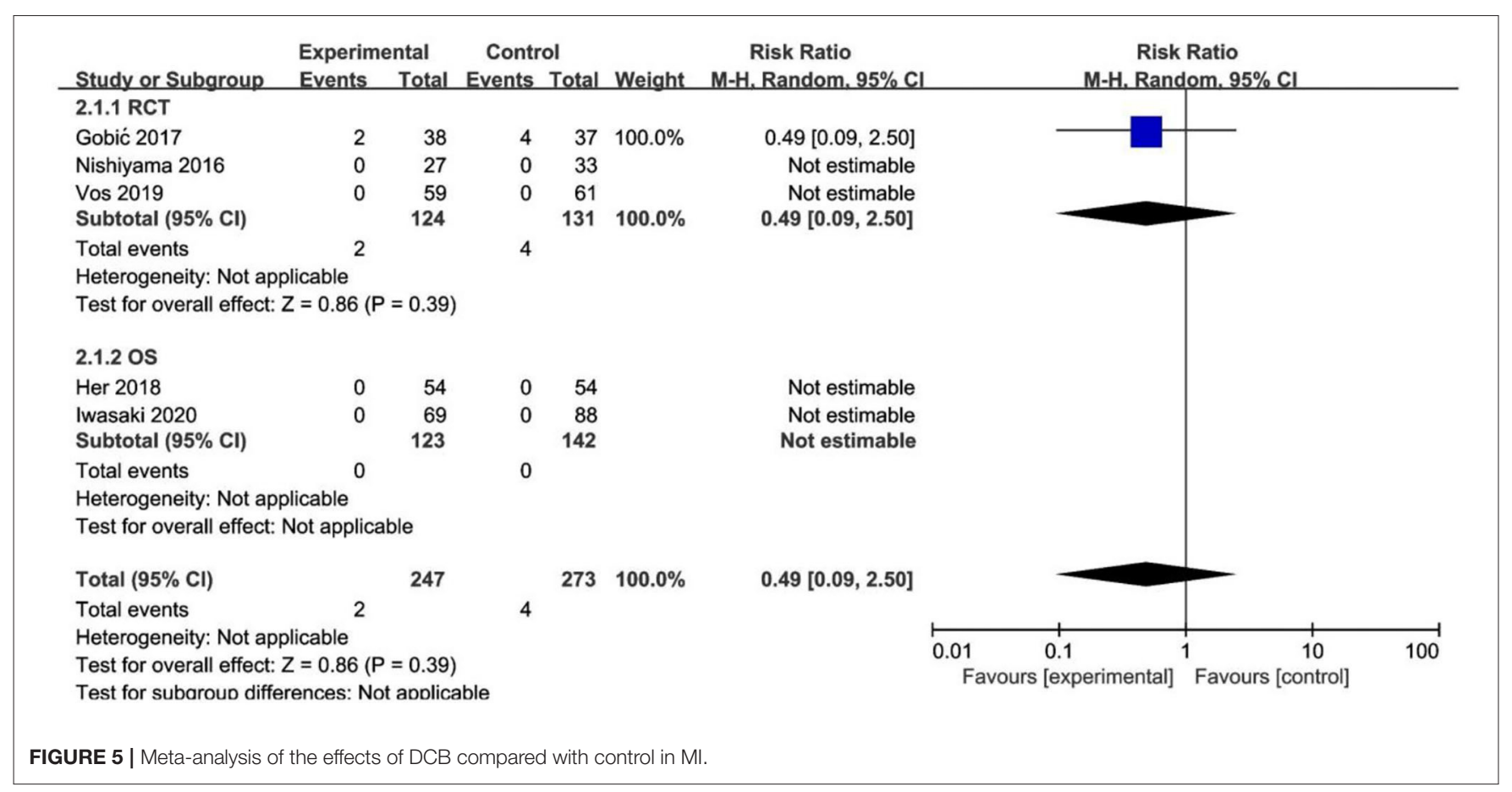

We retrospected the included study but failed to obtain the data of preprocedural MLD. The reasons which were inferred might be that the operators would be more likely to prefer stents than DCB when the target lesions were larger or might be attributed to the treatment allocation itself. LLL was introduced to assess the real changes for the lesions. For LLL, it could be regarded as a mark of unfavorable remodeling, and the mechanism might be inferred as an increase in the plaque area (28). The results showed no significant difference between DCB and stents with significant heterogeneity in RCT. Although the heterogeneity was lower $\left(I^{2}\right.$ from $90 \%$ turned to $58 \%$ ) when the follow-up was more than 6 months according to the subgroup analysis, it still remained a moderate heterogeneity and it was not able to determine the source of heterogeneity. 


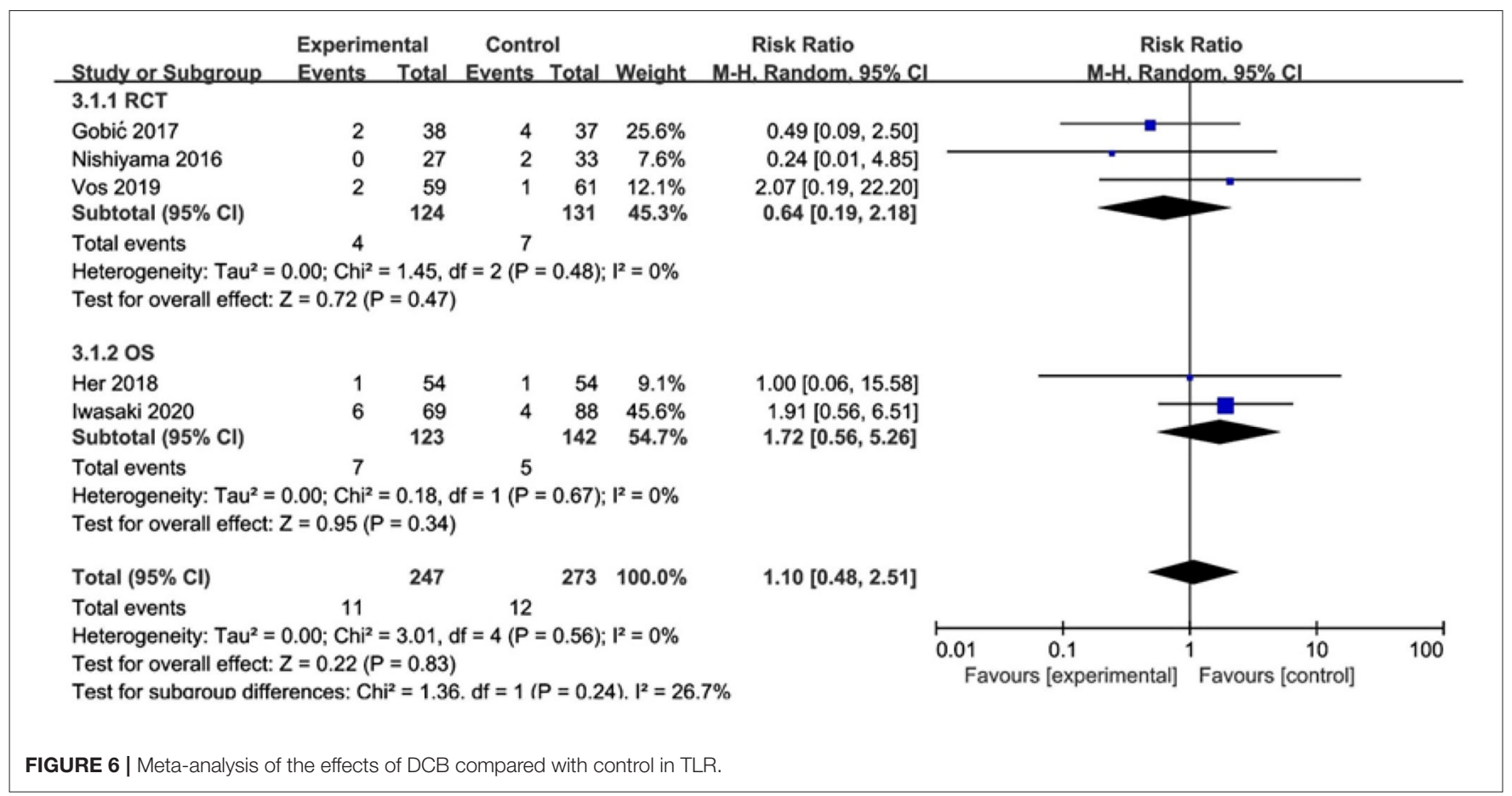

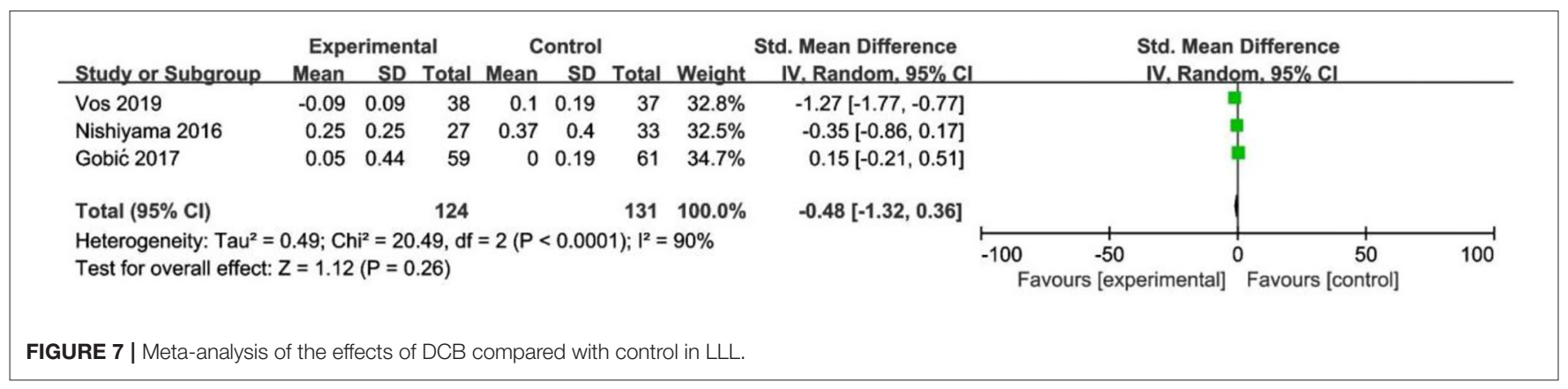

\begin{tabular}{|c|c|c|c|c|c|c|c|c|c|c|c|}
\hline \multirow[b]{2}{*}{ Study or Subgroup } & \multicolumn{3}{|c|}{ Experimental } & \multicolumn{3}{|c|}{ Control } & \multicolumn{2}{|c|}{ Std. Mean Difference } & \multirow{2}{*}{\multicolumn{3}{|c|}{$\begin{array}{l}\text { Std. Mean Difference } \\
\text { IV. Random. } 95 \% \mathrm{Cl}\end{array}$}} \\
\hline & Mean & SD & Total & Mean & SD & Total & Weight & IV. Random. $95 \% \mathrm{Cl}$ & & & \\
\hline Gobić 2017 & 2.39 & 0.37 & 38 & 2.75 & 0.48 & 37 & $28.6 \%$ & $-0.83[-1.31,-0.36]$ & & + & \\
\hline Nishiyama 2016 & 2.36 & 0.46 & 27 & 2.64 & 0.37 & 33 & $23.4 \%$ & $-0.67[-1.19,-0.15]$ & & 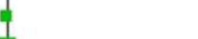 & \\
\hline Vos 2019 & 2.64 & 0.42 & 59 & 2.88 & 0.41 & 61 & $48.0 \%$ & $-0.57[-0.94,-0.21]$ & & 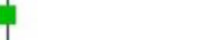 & \\
\hline Total $(95 \% \mathrm{Cl})$ & & & 124 & & & 131 & $100.0 \%$ & $-0.67[-0.92,-0.42]$ & & & \\
\hline \multicolumn{9}{|c|}{ Heterogeneity: $\mathrm{Tau}^{2}=0.00 ; \mathrm{Chi}^{2}=0.72, \mathrm{df}=2(\mathrm{P}=0.70) ; \mathrm{I}^{2}=0 \%$} & $\begin{array}{ll}-100 & -50 \\
\text { Favours [experimental] } & 0\end{array}$ & $\begin{array}{l}0 \\
\text { Favours [control] }\end{array}$ & 100 \\
\hline
\end{tabular}

As the most widely used procedure of PCI, stents are unceasing, updating, and developing. BMS has improved the cardiac hemodynamics though there are a series of complications, such as in-stent restenosis (ISR). The firstgeneration DES, with antiproliferative drugs such as paclitaxel or sirolimus, was investigated and reduced the rate of ISR compared with BMS. However, the late and very late stent thrombosis still exists in the first-generation DES. The dual antiplatelet therapy will also increase the risk of bleeding. To overcome these complications, the second- or newer-generation DES has been investigated with improved stent platforms, biocompatible, durable, or biodegradable polymers, and newer antiproliferative agents, even new technologies, such as ultrathin strut and polymer-free DES, which were developed and assessed by several 


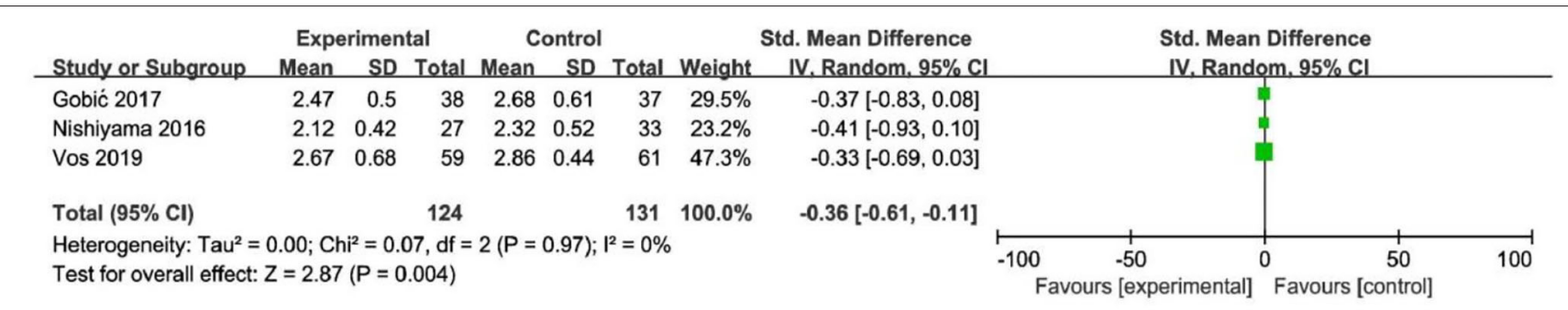

FIGURE 9 | Meta-analysis of the effects of DCB compared with control in MLD2.

TABLE 3 | The subgroup analysis of TLR.

\begin{tabular}{llcccc}
\hline & Group & $\begin{array}{c}\text { The number } \\
\text { of study }\end{array}$ & $\begin{array}{c}\boldsymbol{R} \boldsymbol{R}(\mathbf{9 5 \%} \mathbf{C l}) \\
\mathbf{( 9 5 \%} \mathbf{C l})\end{array}$ & $\boldsymbol{p}$-value & $\boldsymbol{I}^{\mathbf{2} \%}$ \\
\hline The type of & CCAD & 1 & $0.24[0.01,4.85]$ & 0.35 & $\mathrm{NA}$ \\
patient status & STEMI & 2 & $0.78[0.20,2.98]$ & 0.71 & 0 \\
Age & $\leq 60$ years old & 2 & $0.78[0.20,2.98]$ & 0.71 & 0 \\
& $>60$ years old & 1 & $0.24[0.01,4.85]$ & 0.35 & $\mathrm{NA}$ \\
The type of DCB & SeQuent Please & 2 & $0.41[0.10,1.74]$ & 0.23 & 0 \\
& Pantera Lux & 1 & $2.07[0.19,22.20]$ & 0.55 & $\mathrm{NA}$ \\
Follow-up & $\leq 6$ months & 1 & $0.49[0.09,2.50]$ & 0.23 & $\mathrm{NA}$ \\
& $>6$ months & 2 & $0.87[0.11,6.91]$ & 0.89 & $18 \%$ \\
\hline
\end{tabular}

TABLE 4 | The subgroup analysis of LLL.

\begin{tabular}{llcccc}
\hline & Group & $\begin{array}{r}\text { The number } \\
\text { of study }\end{array}$ & $\begin{array}{c}\text { SMD (95\% Cl) } \\
\mathbf{( 9 5 \%} \mathbf{~ C l )}\end{array}$ & $\begin{array}{l}\boldsymbol{p} \text {-value } \\
\boldsymbol{I}^{\mathbf{2} \%}\end{array}$ \\
\hline The type of & CCAD & 1 & $-0.35[-0.86,0.17]$ & 0.18 & $\mathrm{NA}$ \\
patient status STEMI & 2 & $-0.55[-1.94,0.84]$ & 0.44 & $95 \%$ \\
Age & $\leq 60$ years old & 2 & $-0.55[-1.94,0.84]$ & 0.44 & $95 \%$ \\
& $>60$ years old & 1 & $-0.35[-0.86,0.17]$ & 0.18 & $\mathrm{NA}$ \\
The type & SeQuent Please & 2 & $-0.81[-1.72,0.09]$ & 0.08 & $84 \%$ \\
of DCB & Pantera Lux & 1 & $0.15[-0.21,0.51]$ & 0.42 & $\mathrm{NA}$ \\
Follow-up & $\leq 6$ months & 1 & $-1.27[-1.77,-0.77]<0.00001$ & $\mathrm{NA}$ \\
& $>6$ months & 2 & $-0.48[-1.32,0.36]$ & 0.79 & $58 \%$ \\
\hline
\end{tabular}

TABLE 5 | The subgroup analysis of MLD1.

\begin{tabular}{lccccc}
\hline & Group & $\begin{array}{c}\text { The number } \\
\text { of study }\end{array}$ & $\begin{array}{c}\text { Mean } \pm \text { SD } \\
\mathbf{( 9 5 \%} \mathbf{C l})\end{array}$ & $\boldsymbol{p}$-value & $\boldsymbol{I}^{\mathbf{2} \%}$ \\
\hline The type of & CCAD & 1 & $-0.67[-1.19,-0.15]$ & 0.01 & $\mathrm{NA}$ \\
patient status STEMI & 2 & $-0.67[-0.96,-0.38]$ & $<0.00001$ & 0 \\
Age & $\leq 60$ years old & 2 & $-0.67[-0.96,-0.38]$ & $<0.00001$ & 0 \\
& $>60$ years old & 1 & $-0.67[-1.19,-0.15]$ & 0.01 & $\mathrm{NA}$ \\
The type & SeQuent Please & 2 & $-0.76[-1.11,-0.41]$ & $<0.0001$ & 0 \\
of DCB & Pantera Lux & 1 & $-0.57[-0.94,-0.21]$ & 0.002 & $\mathrm{NA}$ \\
\hline
\end{tabular}

trials. Studies showed that the risk of early or late complications was reduced in ACS and showed a better effect in patients with diabetes. Besides, it allowed shorter dual antiplatelet therapy in
TABLE 6 | The subgroup analysis of MLD2.

\begin{tabular}{llcccc}
\hline & Group & $\begin{array}{c}\text { The number } \\
\text { of study }\end{array}$ & $\begin{array}{c}\text { Mean } \pm \text { SD } \\
\mathbf{( 9 5 \%} \mathbf{C l})\end{array}$ & \multicolumn{1}{c}{$\boldsymbol{p}$-value } & $\boldsymbol{I}^{\mathbf{2} \%}$ \\
\hline The type of & CCAD & 1 & $-0.41[-0.93,0.10]$ & 0.12 & NA \\
patient status & STEMI & 2 & $-0.35[-0.63,-0.06]$ & 0.02 & 0 \\
Age & $\leq 60$ years old & 2 & $-0.35[-0.63,-0.06]$ & 0.02 & 0 \\
& $>60$ years old & 1 & $-0.41[-0.93,0.10]$ & 0.12 & NA \\
The type & SeQuent Please & 2 & $-0.39[-0.73,-0.05]$ & 0.02 & 0 \\
of DCB & Pantera Lux & 1 & $-0.33[-0.69,0.03]$ & 0.07 & NA \\
Follow-up & $\leq 6$ months & 1 & $-0.37[-0.83,0.08]$ & 0.11 & NA \\
& $>6$ months & 2 & $-0.36[-0.65,-0.06]$ & 0.02 & 0 \\
\hline
\end{tabular}

patients at high risk of bleeding, avoiding bleeding complications. It could also be applied in more complex lesions, such as left main artery lesions, bifurcation, dissection, and calcification with the help of other devices (29-31). There were also some limitations of DES as mentioned before, and more studies on the newer-generation DES were needed to assess the efficacy and safety. DCB has been gradually applied in clinical, which consists of 3 parts: balloon, antiproliferative drug, and drug carrier (coating). It attaches a layer of drug that can inhibit cell proliferation on the surface of the balloon. When the balloon expands, the drug will be fully contacted with the intima of the coronary artery stenosis in a short time and absorbed by the coronary artery tissue. With the development of research, the technology of the drug coating, balloon, and other aspects have been explored further, so as to improve the biocompatibility of balloon, the antirestenosis effect of coating optimize the control of drug time and release rate to reduce the risk of thrombosis (11-13). In the aspect of antiproliferative drugs, paclitaxel, as a cytotoxic drug, can block mitosis and inhibit the proliferation of vascular smooth muscle cells. It is also highly lipophilic and spreads to the vascular wall easily when contacting with intima, achieving the treatment of vascular stenosis. In clinical practice, paclitaxel-coated balloon (PCB) shows the acute surgical effect, reduces the incidence of related complications, and has a high level of immediate technical performance, sufficient short-term efficacy and safety (32). In addition to paclitaxel, sirolimus and other rapamycin derivatives as a cytotoxic drug can also be used in drug balloons, and relevant 
trials have proved that sirolimus-coated balloon is safe, with a lower incidence of adverse events and the rate of vascular reconstruction $(33,34)$.

\section{LIMITATIONS}

There are also some limitations to our study. First, the number of studies we included is relatively small and the sample size is not large enough, which limited the power to detect the difference between DCB and stent group. Second, it still remains heterogeneity in the results of LLL and the origin of heterogeneity is still unknown though subgroup analysis was carried. It is difficult to discuss the source of heterogeneity with quantitative analysis such as meta-regression limited by the number of included studies. The publication bias had not been carried out since the studies included were less than 10. Third, some major adverse clinical events such as stent thrombosis and major bleeding were not added to this study because of the limited number of studies. Besides, the specific information on concomitant medication and risk factors were not reported in the included studies, and thus, it could not be analyzed for further evaluation. Finally, the difference among DCB technologies fails to analyze since there were limited data on new-generation sirolimus DCB. In this study, the antiproliferative drugs were all paclitaxel though the DCB brands were different. More RCT about new-generation sirolimus DCB are needed to be carried out.

\section{REFERENCES}

1. Khan MA, Hashim MJ, Mustafa H, Baniyas MY, Al Suwaidi SKBM, AlKatheeri $\mathrm{R}$, et al. Global epidemiology of ischemic heart disease: results from the global burden of disease study. Cureus. (2020) 12:e9349. doi: 10.7759/cureus.9349

2. Hoole SP, Bambrough P. Recent advances in percutaneous coronary intervention. Heart. (2020) 106:1380-6. doi: 10.1136/heartjnl-2019-315707

3. Li SM, Li YL, Wang SQ, Bai CC. Progress in the processing technology of coronary stents with micro/nano structures. OPTIK. (2017) 2017:31924. doi: 10.1016/j.ijleo.2017.08.136

4. Madhavan MV, Stone GW. Adverse events beyond 1 year after percutaneous coronary intervention. Curr Opin Cardiol. (2020) 35:687-96. doi: 10.1097/HCO.0000000000000792

5. Sharifi Z, Yazdi MJ, Eshraghi A, Vakili V, Ramezani J. Clinical outcomes and complications of treatment with supraflex stent in patients with coronary artery disease: one-year follow-up. Eur J Transl Myol. (2019) 29:8231. doi: 10.4081/ejtm.2019.8231

6. Nestelberger T, Kaiser C, Jeger R. Drug-coated balloons in cardiovascular disease: benefits, challenges, and clinical applications. Expert Opin Drug Deliv. (2020) 17:201-11. doi: 10.1080/17425247.2020.1714590

7. Shibutani H, Akita Y, Oishi Y, Sueyoshi H, Mukai Y, Yutaka K, et al. The potential hazard of a non-slip element balloon causing distal longitudinal stent deformation: the first clinical experience and in vitro assessment. Cardiol J. (2019) 26:645-52. doi: 10.5603/CJ.a2018.0065

8. Hemu M, Kalra D. Coronary artery pseudoaneurysm: a rare complication of drug-eluting stenting. J Am Coll Cardiol. (2019) 73:2274. doi: 10.1016/S0735-1097(19)32880-3

9. Riku S, Suzuki S, Jinno Y, Tanaka A, Ishii H, Murohara T. Coronary drugeluting stent infection complicated by coronary artery aneurysm and purulent pericarditis: complete resolution without surgery. Can J Cardiol. (2020) 36:967.e1-967.e3. doi: 10.1016/j.cjca.2020.01.007

\section{CONCLUSION}

This systematic review and meta-analysis of RCT showed that DCB might provide a promising way on de novo non-small CAD compared with stents. However, more RCTs are still needed to further prove the benefits of the DCB strategy.

\section{DATA AVAILABILITY STATEMENT}

The original contributions presented in the study are included in the article/supplementary material, further inquiries can be directed to the corresponding author.

\section{AUTHOR CONTRIBUTIONS}

All authors contributed to the study's conception and design. Material preparation, data collection, and analysis were performed by KS and ZL. HW would solve the disagreements between KS and ZL. The first draft of the manuscript was written by KS and all authors commented on previous versions of the manuscript. All authors read and approved the final manuscript.

\section{ACKNOWLEDGMENTS}

This research was supported by the Second Hospital of Dalian Medical University. The assistance of the staff is gratefully acknowledged.

10. Neumann FJ, Sousa-uva M, Ahlsson A, Alfonso F, Banning AP, Benedetto U, et al. 2018 ESC/EACTS Guidelines on myocardial revascularization. Euro Intervention. (2019) 14:1435-534. doi: 10.4244/EIJY19M01_01

11. Jeger RV, Eccleshall S, Wan Ahmad WA, Ge J, Poerner TC, Shin ES, et al. Drug-coated balloons for coronary artery disease: third report of the international DCB consensus group. JACC Cardiovasc Interv. (2020) 13:1391402. doi: 10.1016/j.jcin.2020.02.043

12. Gao L, Chen YD. Application of drug-coated balloon in coronary artery intervention: challenges and opportunities. J Geriatr Cardiol. (2016) 13:90613. doi: 10.11909/j.issn.1671-5411.2016.11.005

13. Meneguz-Moreno RA, Ribamar Costa J Jr, Abizaid A. Drug-coated balloons: hope or hot air: update on the role of coronary DCB. Curr Cardiol Rep. (2018) 20:100. doi: 10.1007/s11886-018-1025-4

14. Megaly M, Rofael M, Saad M, Rezq A, Kohl LP, Kalra A, et al. Outcomes with drug-coated balloons in small-vessel coronary artery disease. Catheter Cardiovasc Interv. (2019) 93:E277-86. doi: 10.1002/ccd.27996

15. Liu W, Zhang M, Chen G, Li Z, Wei F. Drug-coated balloon for de novo coronary artery lesions: a systematic review and trial sequential meta-analysis of randomized controlled trials. Cardiovasc Ther. (2020) 2020:4158363. doi: 10.1155/2020/4158363

16. Li M, Guo C, Lv YH, Zhang MB, Wang ZL. Drug-coated balloon versus drugeluting stent in de novo small coronary vessel disease. Medicine (Baltimore). (2019) 98:e15622. doi: 10.1097/MD.0000000000015622

17. Nishiyama N, Komatsu T, Kuroyanagi T, Fujikake A, Komatsu S, Nakamura $\mathrm{H}$, et al. Clinical value of drug-coated balloon angioplasty for de novo lesions in patients with coronary artery disease. Int J Cardiol. (2016) 222:1138. doi: 10.1016/j.ijcard.2016.07.156

18. Gobić D, Tomulić V, Lulić D, Židan D, Brusich S, Jakljević T, et al. Drug-coated balloon versus drug-eluting stent in primary percutaneous coronary intervention: a feasibility study. Am J Med Sci. (2017) 354:55360. doi: 10.1016/j.amjms.2017.07.005 
19. Vos NS, Fagel ND, Amoroso G, Herrman JR, Patterson MS, Piers LH, et al. Paclitaxel-coated balloon angioplasty versus drug-eluting stent in acute myocardial infarction. JACC Cardiovasc Interv. (2019) 12:16919. doi: 10.1016/j.jcin.2019.04.016

20. Her AY, Kim YH, Garg S, Shin ES. Impact of paclitaxel-coated balloon versus newer-generation drug-eluting stent on periprocedural myocardial infarction in stable angina patients. Coron Artery Dis. (2018) 29:4038. doi: 10.1097/MCA.0000000000000620

21. Iwasaki Y, Koike J, Ko T, Funatsu A, Kobayashi T, Ikeda T, et al. Comparison of drug-eluting stents vs. drug-coated balloon after rotational atherectomy for severely calcified lesions of non-small vessels. Heart Vessels. (2021) 36:18999. doi: 10.1007/s00380-020-01684-Z

22. Lin Y, Sun X, Liu H, Pang X, Dong S. Drug-coated balloon versus drug-eluting stent for treating de novo coronary lesions in large vessels: a meta-analysis of clinical trials. Herz. (2020) 46:269-76. doi: 10.1007/s00059-020-04938-8

23. Moher D, Liberati A, Tetzlaff J, Altman DG, PRISMA Group. Preferred reporting items for systematic reviews and metaanalyses: the PRISMA statement. J Clin Epidemiol. (2009) 62:1006-12. doi: 10.1016/j.jclinepi.2009.06.005

24. Cortese B. The role of optimal lesion preparation for de-novo coronary vessels when a stentless intervention strategy is planned. Minerva Cardioangiol. (2020) 68:51-6. doi: 10.23736/S0026-4725.19.05091-6

25. Bonaventura K, Schwefer M, Yusof AKM, Waliszewski M, Krackhardt F, Steen $\mathrm{P}$, et al. Systematic scoring balloon lesion preparation for drug-coated balloon angioplasty in clinical routine: results of the PASSWORD observational study. Adv Ther. (2020) 37:2210-23. doi: 10.1007/s12325-020-01320-2

26. Bax AM, van Rosendael AR, Ma X, van den Hoogen IJ, Gianni U, Tantawy SW, et al. Comparative differences in the atherosclerotic disease burden between the epicardial coronary arteries: quantitative plaque analysis on coronary computed tomography angiography. Eur Heart J Cardiovasc Imaging. (2021) 22:322-30. doi: 10.1093/ehjci/jeaa275

27. Collet C, Grundeken MJ, Asano T, Onuma Y, Wijns W, Serruys PW. State of the art: coronary angiography. EuroIntervention. (2017) 13:63443. doi: 10.4244/EIJ-D-17-00465

28. Yamamoto T, Sawada T, Uzu K, Takaya T, Kawai H, Yasaka Y. Possible mechanism of late lumen enlargement after treatment for de novo coronary lesions with drug-coated balloon. Int J Cardiol. (2020) 321:307. doi: 10.1016/j.ijcard.2020.07.028
29. Azad MAS, Gul U, Javed A. Everolimus-eluting bioresorbable coronary scaffold in Asian patients: a 2-year follow-up. J Pak Med Assoc. (2020) 70:2281-4. doi: 10.47391/JPMA.1149

30. Kuramitsu S, Sonoda S, Ando K, Otake H, Natsuaki M, Anai R, et al. Drugeluting stent thrombosis: current and future perspectives. Cardiovasc Interv Ther. (2021) 36:158-68. doi: 10.1007/s12928-021-00754-x

31. Spione F, Brugaletta S. Second generation drug-eluting stents: a focus on safety and efficacy of current devices. Expert Rev Cardiovasc Ther. (2021) 19:107-27. doi: 10.1080/14779072.2021.1874352

32. Zhang D, Yang R, Wang S, Dong Z. Paclitaxel: new uses for an old drug. Drug Des Devel Ther. (2014) 8:279-84. doi: 10.2147/DDDT. S56801

33. Alfonso F, Avanzas P. Sirolimus-coated balloons: ready for primetime in real world patients? $J$ Cardiovasc Med. (2021) 22:101-3. doi: 10.2459/JCM.0000000000 001072

34. Basavarajaiah S, Athukorala S, Kalogeras K, Panoulas V, Loku Waduge BH, Bhatia G, et al. Mid-term clinical outcomes from use of Sirolimus coated balloon in coronary intervention; data from real world population. Catheter Cardiovasc Interv. (2020). doi: 10.1002/ccd.28998

Conflict of Interest: The authors declare that the research was conducted in the absence of any commercial or financial relationships that could be construed as a potential conflict of interest.

Publisher's Note: All claims expressed in this article are solely those of the authors and do not necessarily represent those of their affiliated organizations, or those of the publisher, the editors and the reviewers. Any product that may be evaluated in this article, or claim that may be made by its manufacturer, is not guaranteed or endorsed by the publisher.

Copyright (C) 2021 Sun, Liu and Wang. This is an open-access article distributed under the terms of the Creative Commons Attribution License (CC BY). The use, distribution or reproduction in other forums is permitted, provided the original author(s) and the copyright owner(s) are credited and that the original publication in this journal is cited, in accordance with accepted academic practice. No use, distribution or reproduction is permitted which does not comply with these terms. 